\title{
$\mathrm{M}|\mathrm{R}| \mathrm{S}$ Internet Journal Nitride Semiconductor Research
}

\section{Structural Properties of GaN Films Grown by Molecular Beam Epitaxy on Singular and Vicinal 6H-SiC(0001)}

\author{
C. D. Lee ${ }^{1}$, R. M. Feenstra ${ }^{1}$, O. Shigiltchoff ${ }^{2}$, R. P. Devaty ${ }^{2}$ and W. J. Choyke ${ }^{2}$ \\ ${ }^{1}$ Department of Physics, Carnegie Mellon University, \\ ${ }^{2}$ Department of Physics and Astronomy, University of Pittsburgh,
}

(Received Monday, January 14, 2002; accepted Thursday, February 21, 2002)

Gallium nitride films are grown by plasma-assisted molecular beam epitaxy (MBE) on $6 \mathrm{H}-$

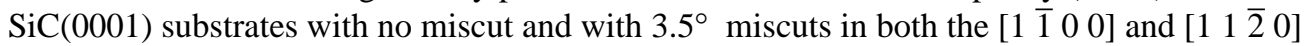
directions. The hydrogen-etched substrates display straight or chevron shaped steps, respectively, and the same morphology is observed on the GaN films. X-ray rocking curves display substantially reduced width for films on the vicinal substrates compared to singular substrates, for the same $\mathrm{Ga} /$ $\mathrm{N}$ flux ratio used during growth.

\section{Introduction}

A number of research groups have in recent years studied the growth using molecular beam epitaxy (MBE) of $\mathrm{GaN}$ on $\mathrm{SiC}$ [1] [2] [3] [4] [5] [6] [7]. Hydrogen etching of the substrates is becoming a standard preparation step for removing polishing damage [8]. Most growth has been performed on singular (no intentional miscut, or on-axis) (0001) Si-face substrates, although a few studies have employed vicinal (miscut, or off-axis) substrates. One report in particular, by Xie et al. [6], has reported significantly improved film quality on the vicinal substrates, as discussed in more detail below.

In this work we report on results for GaN film growth by plasma-assisted MBE on singular $6 \mathrm{H}-$ $\mathrm{SiC}(0001)$ substrates and on vicinal substrates with $\left[\begin{array}{llll}1 & \overline{1} & 0 & 0\end{array}\right]$ and $\left[\begin{array}{llll}1 & 1 & \overline{2} & 0\end{array}\right]$ miscut directions. After H-etching, $\langle 1 \overline{1} 00\rangle$ oriented steps are observed on all types of substrates, independent of miscut direction or magnitude. Subsequent GaN film growth is found to preserve the general morphology of the substrate. For high $\mathrm{Ga} / \mathrm{N}$ flux ratios (where smooth films are achieved), the structural properties of the films grown on the vicinal substrates are found to be substantially better than for those films grown on the singular substrates.

\section{Experimental}

The $6 \mathrm{H}$ polytype of $\mathrm{SiC}$ was used for all the studies reported here. The on-axis $\mathrm{SiC}$ and the substrates miscut $3.5^{\circ}$ towards $\left[\begin{array}{llll}1 & 1 & \overline{2} & 0\end{array}\right]$ were $\mathrm{n}$-type material, purchased commercially from Cree, Inc. The substrates misoriented towards $\left[\begin{array}{llll}1 & \overline{1} & 0 & 0\end{array}\right]$ were cut from a boule of n-type material. Samples were cut at an angle of $3.5 \pm 0.5^{\circ}$ from the $\left[\begin{array}{llll}0 & 0 & 0 & 1\end{array}\right]$ direction towards $\left[\begin{array}{llll}1 & 1 & 0 & 0\end{array}\right]$ using a goniometer accurate to $\pm 0.5^{\circ}$. X-ray Laue patterns were taken to verify the angle. These samples were polished with 90 , $30,15,6,3,1,0.5$ and finally $0.25 \mu \mathrm{m}$ diamond paste. The last four stages took about 20-25 hours each. No visible scratches on the resulting surfaces were detected using the naked eye. Hydrogen etching of the $\mathrm{SiC}$ was performed at $1600-1700^{\circ} \mathrm{C}$ and 1 atm pressure to remove polishing damage [8]. Following transfer of the substrates to the MBE system, Si deposition followed by annealing was done to remove surface oxide and obtain a surface with well-defined structure and stoichiometry, namely, the $\sqrt{ } 3 \times \sqrt{3}-\mathrm{R} 30^{\circ}$ reconstruction containing $1 / 3$ monolayer (ML) of Si adatoms on the $\mathrm{Si}$-face $\mathrm{SiC}(0001)$ surface. This substrate was then taken to the growth temperature and deposition of the $\mathrm{GaN}$ was performed. Our GaN films have Ga polarity [9].

$\mathrm{GaN}$ films of thickness near $1 \mu \mathrm{m}$ were grown at temperatures of $730-800^{\circ} \mathrm{C}$ using various $\mathrm{Ga} / \mathrm{N}$ flux ratios [10]. As discussed previously, as the Ga flux exceeds some critical value the growth morphology as viewed by reflection high-energy electron diffraction (RHEED) goes through a rough to smooth transition [11]. We define an effective $\mathrm{N}$ flux such that the $\mathrm{Ga} / \mathrm{N}$ flux ratio is unity at this transition point. (In terms of actual fluxes, this effective flux is given by $\mathrm{N}_{\mathrm{i}^{-}}$ $\mathrm{N}_{\mathrm{d}}{ }^{*}+\mathrm{Ga}_{\mathrm{d}} *$ where $\mathrm{N}_{\mathrm{i}}$ is the total incident $\mathrm{N}$ flux, $\mathrm{N}_{\mathrm{d}} *$ is the desorbed $\mathrm{N}$ flux at the transition point, and $\mathrm{Ga}_{\mathrm{d}}{ }^{*}$ is the desorbed Ga flux at the transition point). Films were 
characterized using atomic force microscopy (AFM) and x-ray rocking curves, the latter performed in a triple-axis configuration. In most cases the AFM was performed in contact mode, although for the $\mathrm{SiC}$ miscut towards $\left[\begin{array}{llll}1 & 1 & 2 & 0\end{array}\right]$ significant surface charging problems were observed and noncontact (tapping) mode was needed to overcome them.

\section{Results \& Discussion}

\subsection{Morphology of $\mathrm{H}$-etched $\mathrm{SiC}$ substrates}

Our $6 \mathrm{H}-\mathrm{SiC}(0001)$ substrates are prepared by $\mathrm{H}$-etching, as illustrated in Figure 1. Figures 1(a) and (b) review results for singular substrates [8]: The morphology of as-received substrates [Figure 1(a)] displays many polishing scratches. Following etching, full-unitcell height (15 ̊) steps, arising from an unintentional miscut of the substrate, are seen on the surface [Figure 1(b)]. On a larger scale the surface is found to break into low-angle facets, with neighboring facets having a different $\left\langle\begin{array}{llll}1 & 1 & 0 & 0\end{array}\right.$ step direction and the angle of each facet being less than the overall miscut of the wafer.

Results for a $\mathrm{H}$-etched substrate miscut by $3.5^{\circ}$

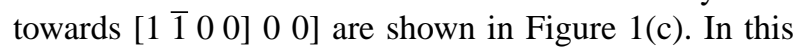
case the surface is seen to consist of a series of $\approx 300$ $\mathrm{nm}$ wide strips, with normal vector oriented along the miscut direction. These strips are separated by step bunches, typically $3 \mathrm{~nm}$ high. In contrast, results for a $\mathrm{H}$-etched substrates miscut by $3.5^{\circ}$ towards $\left[\begin{array}{llll}1 & 1 & \overline{2} & 0\end{array}\right]$ are shown in Figure 1(d). We now observe half-unit-cell height $(7.5 \AA)$ steps separated by $\approx 12 \mathrm{~nm}$ wide terraces. The steps, on average, have normal vector oriented in the miscut direction. However, it is clearly seen in Figure 1(d) that on a small scale the steps have a different orientation, i.e. the step edges have a zig-zag or chevron morphology. The precise orientation of this small-scale structure of the step edges is difficult to discern from Figure 1(d), but we believe the normal vectors to be

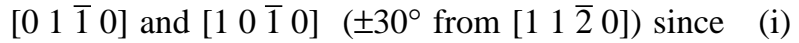
this is a low energy step orientation for $\mathrm{SiC}$ steps [8], and (ii) the same step orientation is seen on GaN films grown on these substrates as shown below.

\subsection{GaN films on H-etched SiC}

Before discussing results for GaN films grown on vicinal substrates, we first review results for growth on singular material as shown in Figure 2 [7]. For samples grown with $\mathrm{Ga} / \mathrm{N}$ ratio only slightly greater than unity, the surface is covered with pits [Figure 2(a)]. The pits are formed with facetted sidewalls and flat-topped (0001) oriented ridges, as illustrated by the linecut in Figure 2(a). From AFM linescans the sidewalls are found to have an angle of $30^{\circ} \pm 5^{\circ}$ relative to the (0001) surface. From facet-derived features in RHEED patterns this angle is found more precisely to be $33^{\circ} \pm 2^{\circ}$. The facets thus correspond to $\{10 \overline{1} 3\}$ planes, which have an angle of $32.0^{\circ}$ relative to the (0001) surface. This result is similar to that of Heying et al. who report $\{10$ onebar3 $\}$ or $\{10 \overline{1} 4\}$ facets [12]. An analysis of facet angles in the AFM image (of the type done e.g. in Ref. [13]) reveals that all six equivalent $\{10 \overline{1} 3\}$ facets are present in approximately equal amounts on the surface. As the $\mathrm{Ga} / \mathrm{N}$ ratio is increased to about 1.1 fewer pits appear on the surface, and those which are present tend to merge into trenches which separate plateaus of atomically flat morphology [Figure 2(b)]. For $\mathrm{Ga} / \mathrm{N}$ ratios near 1.3, isolated pits with typical separation of about 1 $\mu \mathrm{m}$ are seen in the morphology [Figure 2(c)]. TEM studies reveal that these pits are associated with dislocations intersecting the surface [7] [14]; some type of preferential film decomposition or reduced growth rate apparently occurs at these points. Finally, for growth under very $\mathrm{Ga}$-rich conditions, $\mathrm{Ga} / \mathrm{N}$ ratio above about 1.5 , the morphology is flat with only monatomic steps revealed in AFM [Figure 2(d)]. On a large scale, the GaN film morphology follows that of the $\mathrm{SiC}$, with low-angle facets on the surface [10]. Gallium droplets are observed on these surfaces grown with high $\mathrm{Ga} / \mathrm{N}$ ratios, in agreement with the observations of Heying et al. [12].

As shown in Figure 2, the roughness of films grown with $\mathrm{Ga} / \mathrm{N}$ flux ratios near unity is significantly greater than for those with higher $\mathrm{Ga} / \mathrm{N}$ ratios. Rms roughness values are 35, 20, 4, and $3 \mathrm{~nm}$ for the images shown in Figs. 2(a)-(d) respectively. However, for these singular substrates, we find that even though the morphology at low $\mathrm{Ga} / \mathrm{N}$ flux ratio is worse (i.e. rougher) than that at high $\mathrm{Ga} / \mathrm{N}$ flux ratio, the structural properties of the lowflux-ratio films is much improved. In particular, the FWHM of asymmetric (1120) x-ray rocking curves are about $2 \times$ less for films with flux ratio near unity compared to that of films with flux ratio of $1.3-1.5$ [14]. TEM reveals an order-of-magnitude reduction in dislocation density for the low-flux-ratio films. The mechanism for this improvement in film quality is found to be the tendency of dislocations to cluster near the topographic minima, where their probability of annihilation is increased due to their closer proximity [14].

The surface morphology of our GaN films grown on vicinal $\mathrm{SiC}$ is pictured in Figure 3. These results can be seen to reflect the morphology of the underlying $\mathrm{SiC}$ substrates. The films grown on a substrate miscut towards $\left[\begin{array}{llll}1 & 1 & 0 & 0\end{array}\right]$ display similar size terraces (with $\left[\begin{array}{llll}1 & 1 & 0 & 0\end{array}\right]$ normal vectors) as the substrate, although these $\mathrm{GaN}$ strips are not completely continuous along their length [Figure 3(a)]. The films grown on the substrate miscut towards $\left[\begin{array}{llll}1 & 1 & \overline{2} & 0\end{array}\right]$ display steps with average orientation having [ $\left[\begin{array}{llll}1 & 1 & \overline{2} & 0\end{array}\right]$ normal, but with $\left[\begin{array}{llll}0 & 1 & \overline{1} & 0\end{array}\right]$ and $\left[\begin{array}{llll}1 & 0 & 1 & 0\end{array}\right]$ oriented step edges seen on a smaller scale [Figure 3(b)]. Note however that these features are much 
larger than the corresponding ones seen in Figure 1(d), indicating significant step bunching and terrace growth during the GaN film growth. Rms roughness is $3 \mathrm{~nm}$ for both Figure 3 (a) and (b), similar to the values found for Figure 2 (c) and (d). In all cases those films are probably smooth enough so as to not to produce any deleterious effects in device processing or operation (e.g. room temperature mobility values in field-effect transistors would generally not be affected by this level of surface or interface roughness, although low temperature mobility values may be impacted [15]).

$\mathrm{X}$-ray results for our films are listed in Table 1 . We report FWHM of rocking curves acquired in both symmetric (0002) and asymmetric (10 $\overline{1} 2)$ configurations. The former reflects the screw dislocation density in the material and the latter reflects a combination of screw and edge dislocation densities. Actually, since in our material the edge dislocation density is 1-2 orders of magnitude larger than the screw dislocation density (and there are very few dislocations of mixed character), the (1012) width reflects primarily the edge dislocation density. It is this (10 $\overline{1} 2)$ width which is the important parameter in Table 1 for distinguishing the various films.

The first two entries in Table 1 list films grown on on-axis $\mathrm{SiC}$ with $\mathrm{Ga} / \mathrm{N}$ ratio near unity. These films have

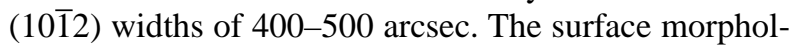
ogy for those films is somewhat rough, as described above. The next three entries in Table 1 list films grown

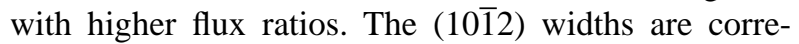
spondingly larger, for the reason discussed in the previous section. The final four entries in Table 1 give results for growth on the vicinal substrates, for $\mathrm{Ga} / \mathrm{N}$ ratios of 1.5. We find that the $(10 \overline{1} 2)$ widths for these films are significantly less than those for the films grown on singular substrates using the same flux ratios. In fact, the results for the films grown on vicinal substrates are comparable to those obtained for our best (i.e. low-fluxratio) growth on the singular substrates, whereas the morphology of the growth on the vicinal substrates as seen in Figure 3 is improved compared to that on those low-flux-ratio films on the singular substrates.

For several of the films grown on miscut substrates listed in Table 1 we measured the x-ray rocking curves for two inequivalent orientations of the vicinal substrates relative to the incident beam. For [ $\left[\begin{array}{llll}1 & 1 & \overline{2} & 0\end{array}\right]$ or $\left[\begin{array}{ll}0 \\ 0\end{array}\right.$ $1 \overline{1} 0$ ] miscut directions the first value listed in Table 1 corresponds to a rocking curve scan with in-plane component perpendicular or parallel, respectively, to the miscut direction. The second value listed in Table 1 then corresponds to a scan direction with in-plane component rotated by $60^{\circ}$ relative to the first. Not surprisingly some variation in the width is seen, implying that the mechanism for the dislocation reduction in these films does indeed involve some specific aspect of their vici- nality. TEM measurements have not been performed to date, so no further information in available concerning the mechanism for dislocation reduction in these films. Recently Xie et al. [6] have reported results for dislocation reduction in $\mathrm{GaN}$ films grown on vicinal $\mathrm{SiC}$. In their case the initial $\mathrm{SiC}$ substrates were not $\mathrm{H}$-etched, so that their screw dislocation density for growth on singular substrates was very high. This value was greatly reduced for the growth on vicinal substrates, which they interpret as being due to nucleation, growth and coalescence of $\mathrm{GaN}$ islands at step edges rather than on terraces [6]. They also observed a reduction in edge dislocation density, similar to that reported here, although no specific mechanism was suggested for that reduction. Their results are thus consistent with those reported here, although their model for the reduction in screw dislocation density is not relevant for our situation of H-etched substrates in which our screw dislocation density is low for both singular and vicinal substrates.

\section{Summary}

In summary, we have grown $\mathrm{GaN}$ films by plasmaassisted MBE on singular $6 \mathrm{H}-\mathrm{SiC}(0001)$ substrates and on vicinal substrates with [ $\left[\begin{array}{llll}1 & \overline{1} & 0 & 0\end{array}\right]$ and $\left[\begin{array}{llll}1 & 1 & \overline{2} & 0\end{array}\right]$ miscut directions. X-ray rocking curves display substantially reduced width for films on the vicinal substrates compared to singular substrates, for the same $\mathrm{Ga} / \mathrm{N}$ flux ratio used during growth. Films grown on vicinal substrates at high $\mathrm{Ga} / \mathrm{N}$ flux ratio have rocking curve widths comparable to those obtained for our best growth on singular substrates (i.e. using low $\mathrm{Ga} / \mathrm{N}$ ratio, near unity), but the roughness for those films on vicinal substrates is about an order-of-magnitude lower than that of the low-fluxratio films on singular substrates.

\section{ACKNOWLEDGMENTS}

This work was supported the Office of Naval Research (grant N00014-96-1-0214, monitored by C. Wood).

\section{REFERENCES}

[1] V Ramachandran, AR Smith, RM Feenstra, J. Vac. Sci. Technol. A 17, 1289-1293 (1999).

[2] V. M. Torres, J. L. Edwards, B. J. Wilkens, D. J. Smith, R. B. Doak, I. S. T. Tsong, Appl. Phys. Lett. 74, 985 (1999).

[3] Q. Z. Xue, Q. K. Xue, Y. Hasegawa, I. S. T. Tsong, T. Sakurai, Appl. Phys. Lett. 74, 2468 (1999).

[4] R. Lantier, A. Rizzi, D. Guggi, H. Luth, B. Neubauer, D. Gerthsen, S. Frabboni, G. Coli, R. Cingolani, MRS Internet J. Nitride Semicond. Res. 4S1, G3.50 (1999).

[5] O. Brandt, R. Muralidharan, P. Waltereit, A. Thamm, A. Trampert, H. von Kiedrowski, K. H. Ploog, Appl. Phys. Lett. 75, 4019 (1999).

[6] M. H. Xie, L. X. Zheng, S. H. Cheung, Y. F. Ng, H. Wu, S. Y. Tong, N. Ohtani, Appl. Phys. Lett. 77, 1105 (2000). 
[7] C. D. Lee, V. Ramachandran, A. Sagar, R. M. Feenstra, D. W. Greve, W. L. Sarney, L. Salamanca-Riba, D. C. Look, S. Bai, W. J. Choyke, R. P. Devaty, J. Electron. Mater. 30, 162 (2001).

[8] V Ramachandran, MF Brady, AR Smith, RM Feenstra, DW Greve, J. Electron. Mater. 27, 308-312 (1998).

[9] A. R. Smith, R. M. Feenstra, D. W. Greve, M. S. Shin, M. Skowronski, J. Neugebauer, J. E. Northrup, Appl. Phys. Lett. 72, 2114-2116 (1998).

[10] C. D. Lee, A. Sagar, R. M. Feenstra, W. L. Sarney, L. Salamanca-Riba, J. W. P. Hsu, Phys. Stat. Sol. A 188, 595 (2001).

[11] A. R. Smith, V. Ramachandran, R. M. Feenstra, D. W. Greve, A. Ptak, T. Myers, W. Sarney, L. Salamanca-Riba,
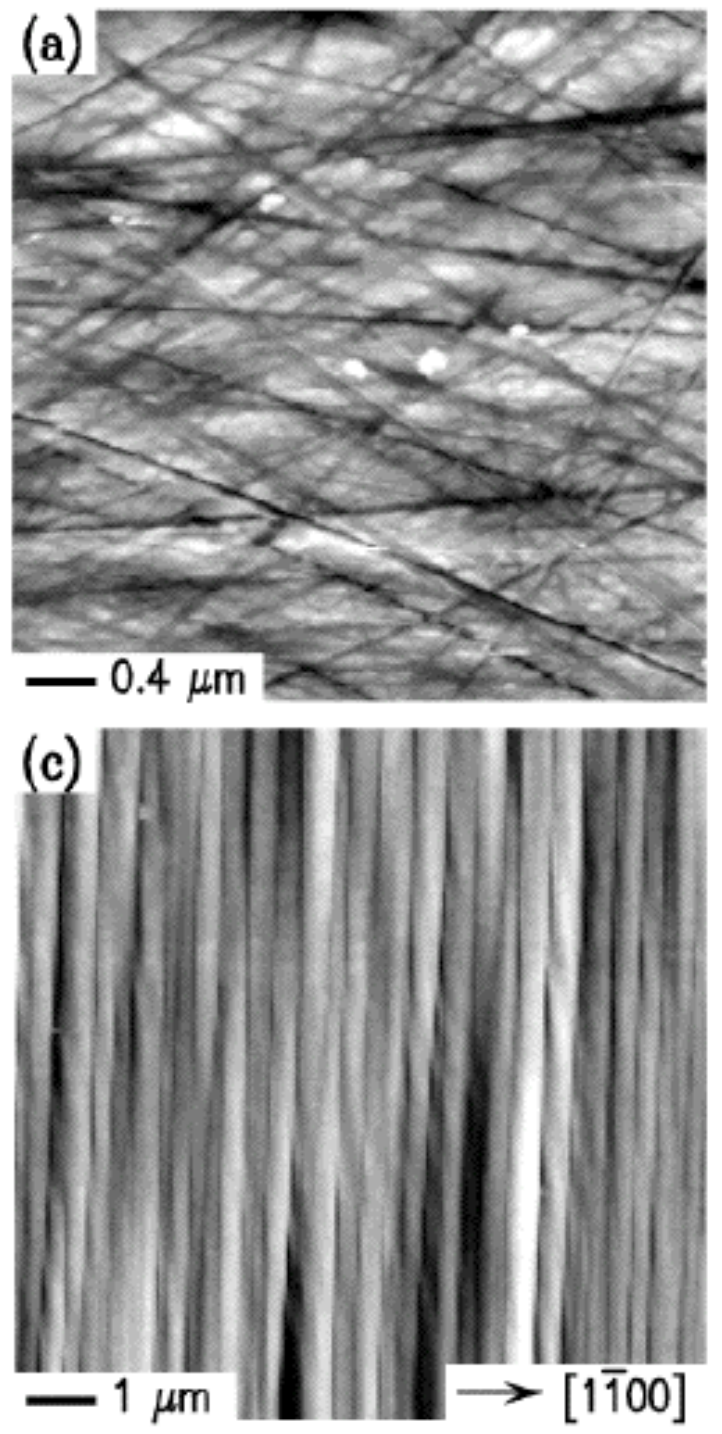

M. Shin, M. Skowronski, MRS Internet J. Nitride Semicond. Res. 3, 12 (1998).

[12] B. Heying, R. Averbeck, L. F. Chen, E. Haus, H. Riechert, J. S. Speck, J. Appl. Phys. 88, 1855 (2000).

[13] M. A. Lutz, R. M. Feenstra, P. M. Mooney, J. Tersoff, J. O. Chu, Surf. Sci. Lett 316, L1075 (1994).

[14] C. D. Lee, A. Sagar, R. M. Feenstra, C. K. Inoki, T. S. Kuan, W. L. Sarney, L. Salamanca-Riba, Appl. Phys. Lett. 79, 3248 (2001).

[15] D. Monroe, Y. H. Xie, E. A. Fitzgerald, P. J. Silverman, G. P. Watson, J. Vac. Sci. Technol. B 11, 1731 (1993).

\section{FIGURES}
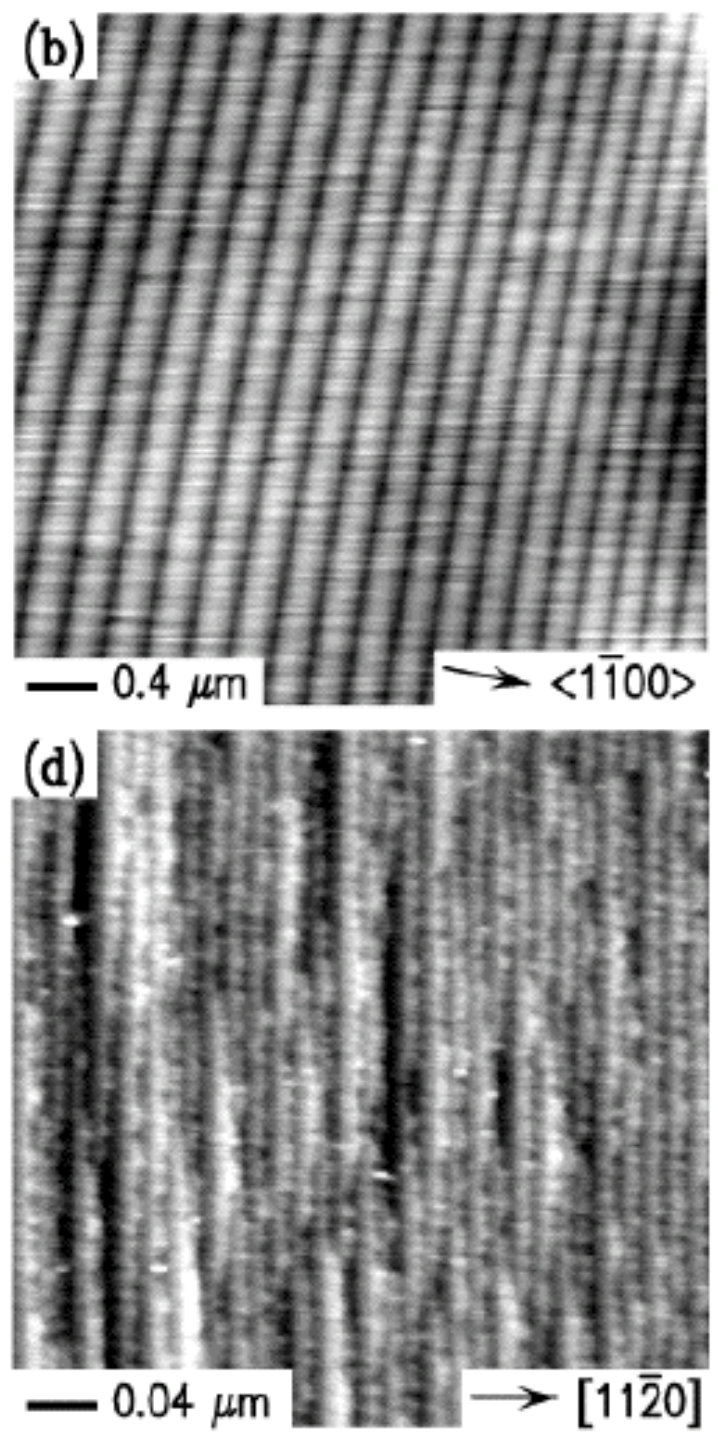

Figure 1. AFM images of $6 \mathrm{H}-\mathrm{SiC}(0001)$ surfaces: (a) on-axis, as-received; (b) on-axis, H-etched; (c) off-axis, $3.5^{\circ}$ towards [1 [ $\left.\begin{array}{llll}1 & 1 & 0 & 0\end{array}\right]$, H-etched; (d) off-axis, $3.5^{\circ}$ towards $\left[\begin{array}{llll}1 & 1 & 2 & 0\end{array}\right]$, H-etched. Gray scale ranges are (a) $10 \mathrm{~nm}$, (b) $0.7 \mathrm{~nm}$, (c) $7 \mathrm{~nm}$, and (d) 1 $\mathrm{nm}$. Note the varying lateral scales. 

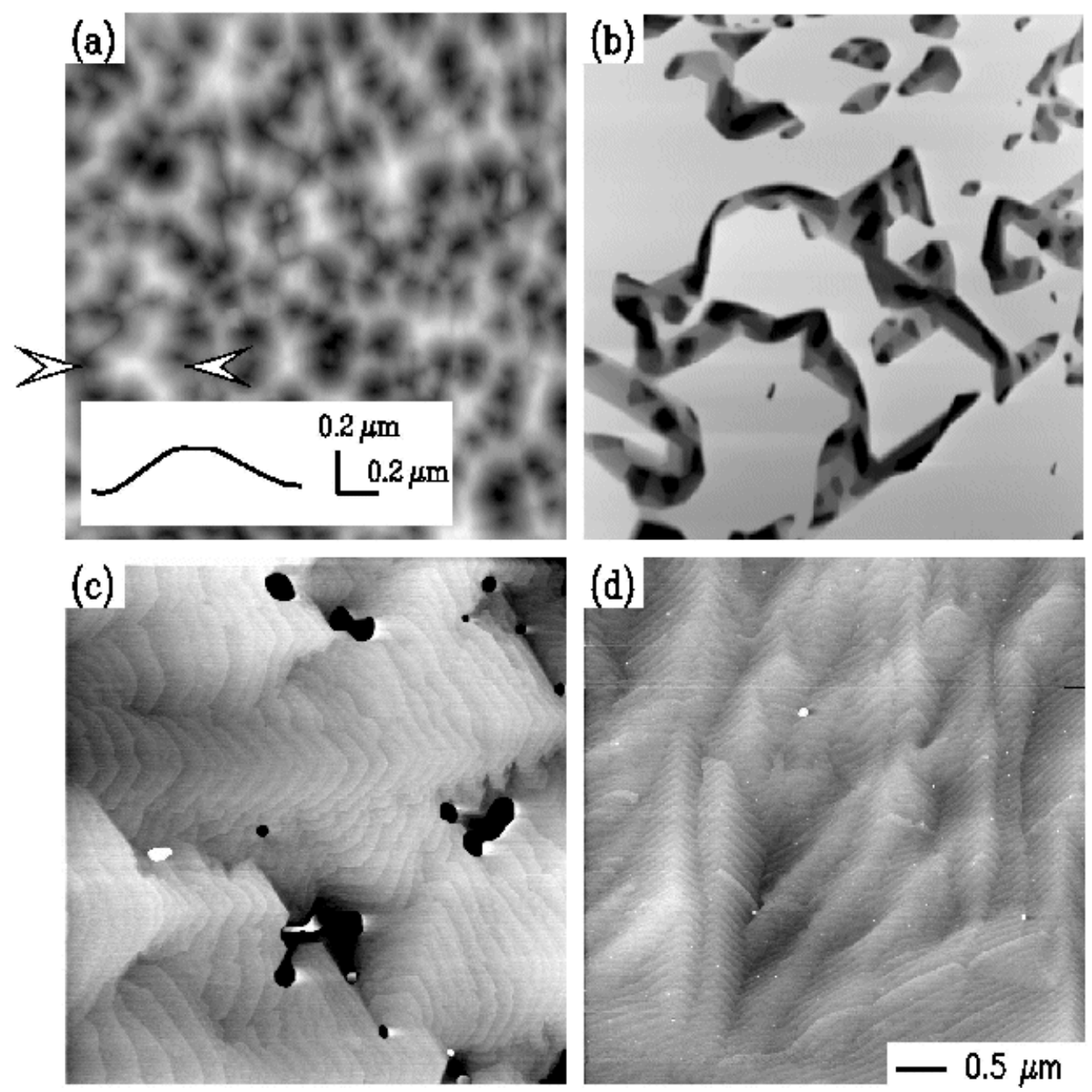

Figure 2. AFM images of $\mathrm{GaN}(0001)$ films grown on $\mathrm{H}$-etched $6 \mathrm{H}-\mathrm{SiC}$, with $\mathrm{Ga} / \mathrm{N}$ flux ratios of (a) 1.05 , (b) 1.1 , (c) 1.3 , and (c) 1.6. The gray scale ranges are $210 \mathrm{~nm}, 86 \mathrm{~nm}, 5 \mathrm{~nm}$, and $5 \mathrm{~nm}$ for (a)-(d) respectively. The film pictured in (a) was grown at $800^{\circ} \mathrm{C}$ and the other films were grown at $750^{\circ} \mathrm{C}$. In (a), a line cut taken between the arrows is shown in the inset. 

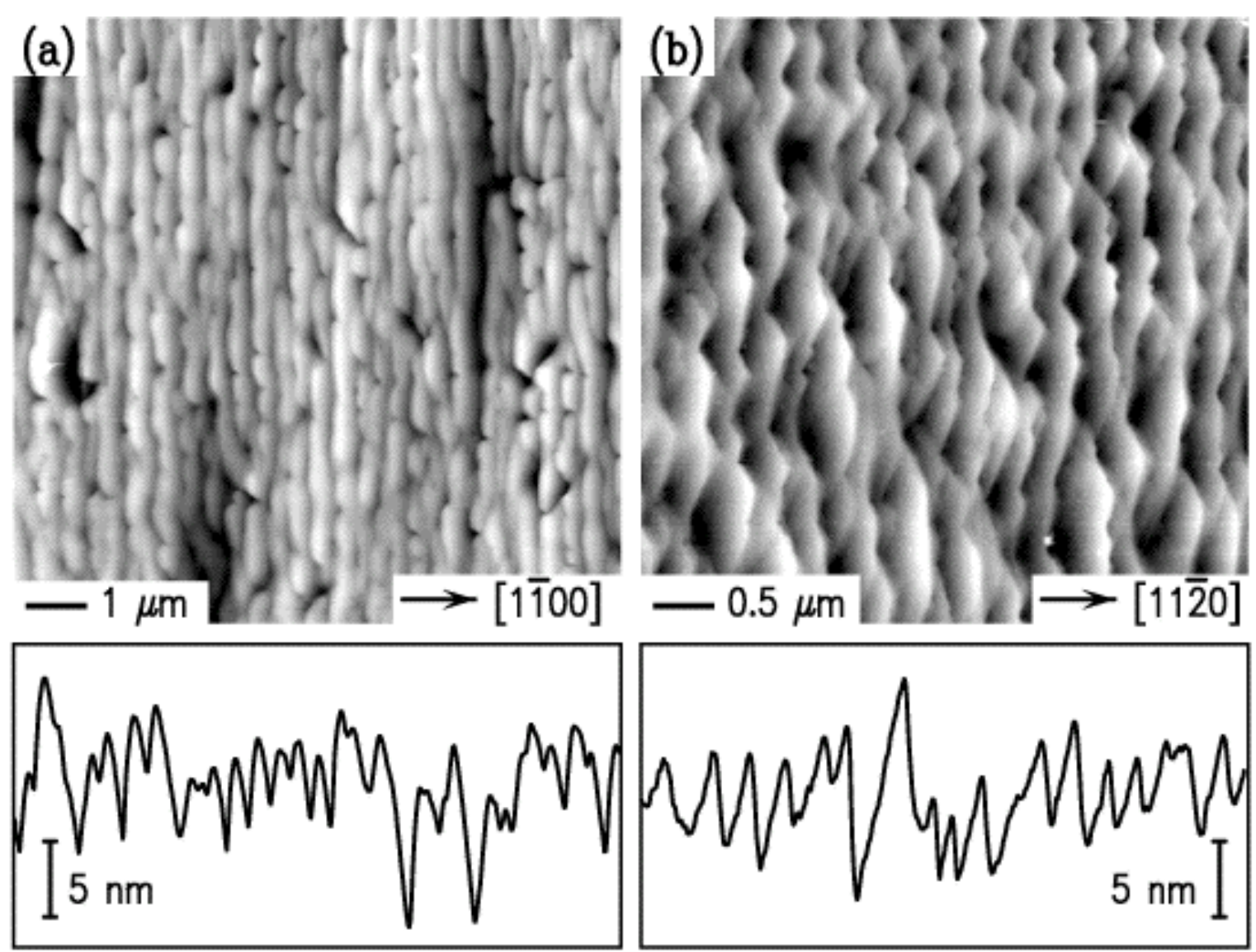

Figure 3. AFM images of $\mathrm{GaN}(0001)$ films grown on $\mathrm{H}$-etched miscut $\mathrm{SiC}$, with $\mathrm{Ga} / \mathrm{N}$ ratio of 1.5 for both films. Substrates used

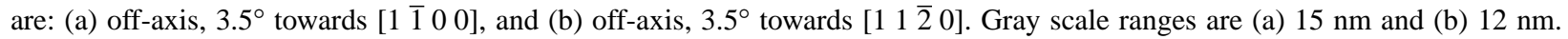
Linescan profiles taken across the middle of each image are shown below the images (a linear background subtraction has been applied to all images and linescans).

\section{TABLES}

Table 1. X-ray rocking curve FWHM values for GaN films of specified miscut, growth temperature, Ga/N ratio, and thickness. Two values for the FWHM of films on miscut substrates refer to different sample orientations as discussed in the text.

\begin{tabular}{|l|l|l|l|l|l|}
\hline Miscut & $\mathrm{T}\left({ }^{\circ} \mathrm{C}\right)$ & Ga/N ratio & thickness $(\mu \mathrm{m})$ & \multicolumn{2}{l|}{ FWHM X-ray rocking curves $(\operatorname{arcsec})$} \\
\hline & & & & $(0002)$ & $(10 \overline{1} 2)$ \\
\hline On-axis & 730 & 1.1 & 1.0 & 121 & 500 \\
\hline On-axis & 780 & 1.1 & 2.0 & 119 & 410 \\
\hline On-axis & 750 & 1.4 & 1.3 & 128 & 927 \\
\hline On-axis & 750 & 1.7 & 1.0 & 57 & 960 \\
\hline On-axis & 730 & 1.9 & 1.0 & 218 & 960 \\
\hline $3.5^{\circ}$ towards $<11-20>$ & 750 & 1.5 & 1.0 & 68 & 520 \\
\hline $3.5^{\circ}$ towards $<11-20>$ & 750 & 1.5 & 1.0 & 135 & $442 / 575$ \\
\hline $3.5^{\circ}$ towards $<1-20>$ & 750 & 1.5 & 1.2 & 115 & $490 / 634$ \\
\hline $3.5^{\circ}$ towards $<1-100>$ & 750 & 1.5 & 1.0 & 93 & $612 / 418$ \\
\hline
\end{tabular}

